\title{
AProSiMA - Um ambiente na web para resolução cooperativa de problemas baseado em simulação multiagente
}

\author{
Lívia Lopes Azevedo ${ }^{1,2}$, \\ Crediné Silva de Menezes ${ }^{2}$ \\ ${ }^{1}$ Instituto de Ciências e Letras do Médio Araguaia (ICLMA) - UFMT \\ ${ }^{2}$ Centro Tecnológico - PPGEE - Universidade Federal do Espírito Santo (UFES) \\ livialcpd.ufmt.br, credinelinf.ufes.br
}

\begin{abstract}
Resumo.
Neste artigo apresentamos o arcabouço conceitual do ambiente para resolução cooperativa de problemas baseado em simulação multiagente, denominado AProSiMA, um ambiente projetado para web que além de oferecer recursos para busca de informações elou disponibilizar o problema para que outros usuários do ambiente possam cooperar, disponibiliza uma ferramenta para construção de modelos de simulação baseado em multiagente. $O$ ambiente permite a construção cooperativa de modelos, viabiliza a discussão sobre os modelos construídos e permite a manutenção de diferentes versões de um mesmo modelo. Destacamos que o processo de construção do modelo emprega os mesmos passos dos métodos de resolução cooperativa de problemas, sendo as duas abordagens suportadas pelo ambiente. O ambiente está sendo construído visando o uso didático para exploração de sistemas descentralizados no contexto do ensino fundamental.
\end{abstract}

Palavras-chave: Resolução de problemas, ambientes cooperativos, simulação multiagente.

\begin{abstract}
.
In this article we present the conceptual outline of the environment for cooperative problems solving based on multiagent simulation, called - AProSiMA, an environment projected for web that offers resources for searching information and/or make available the problem, so that other users of the environment can cooperate, and it makes available a tool for building of multiagent based simulation models. The environment allows the cooperative building of models, it makes possible the discussion on the built models and it allows the maintenance of different versions of one same model. We highlight that the process of the model's building uses the same steps of the methods of cooperative problems solving, being the two approaches supported by the environment. The environment is being built aiming the didactic use for exploration of decentralized systems in the basic education context.
\end{abstract}

Keywords: Problems solving, cooperative environments, multiagent simulation

\section{Introdução}

Muitas de nossas atividades diárias envolvem algum tipo de resolução de problemas. Por exemplo, planejar o controle da gripe aviária, tomar medidas para superação de um acidente ambiental, decidir como estruturar um artigo sobre uma pesquisa científica, como trocar o pneu de um carro, fazer planos para uma viagem de férias, como organizar os livros numa estante, e assim por diante. Podemos deparar com problemas 
nunca visto antes, e também com problemas que são bastante familiares. Naturalmente, cada problema exige uma estratégia de solução apropriada. Muitos problemas podem ser resolvidos com relativa facilidade e não exigindo esforço. Outros já exigem um esforço mental bem maior, possivelmente requerendo a elaboração de um plano de execução, testar algumas hipóteses, um processamento e, provavelmente, muita análise. Há problemas cujos objetivos são óbvios, enquanto em outros estes estão bastante obscurecidos. Em alguns problemas o número de variáveis é simples de controlar, seus objetivos são determinísticos, a busca pela solução encontra num conhecimento específico e um único resolvedor pode dar conta de resolvê-lo. Entretanto, há uma classe de problemas oposta a todos esses elementos citados, são problemas que requerem uma abordagem interdisciplinar e uma base de conhecimento diversificada, denominados de problemas complexos. Para resolver problemas dessa classe é necessário o esforço conjunto de especialistas em domínio específico, fazendo com que um fator adicional cresça em importância: a cooperação.

Da mesma forma que existe níveis de dificuldades para os problemas, há também categorização para sua resolução. Dois principais tipos são reconhecidos na pesquisa sobre resolução de problemas, segundo (Rubinstein, apud Deek 1977) são problemas de análise e problemas de síntese, e também a combinação de ambos. $\mathrm{O}$ problema de análise, também conhecido como problemas de transformação por (Greeno, 1978) e regressão por (Polya, 1995) - é caracterizado pelo processo típico de estabelecer um plano, a partir da incógnita (ou da conclusão) e caminhar no sentido dos dados (ou hipótese). Para resolver esse tipo de problema Polya sugere o raciocínio regressivo ou raciocínio pra trás. Os problemas de síntese ou problema de arranjo para (Greeno, 1978) e resolução construtiva para (Polya, 1995) - são aqueles em que o plano de ação começa daquilo que é conhecido, é o processo contrário ao da análise. Para buscar a solução, Polya sugere o raciocínio progressivo.

Muitas metodologias de resolução de problemas têm sido desenvolvidas com o objetivo de conduzir à resolução de problema. Associado a elas, desde os primeiros tempos, os homens também têm criado modelos como uma forma de representar, explicar e entender os fenômenos em estudo (naturais, sociais, químicos, físicos, etc.) objetivando encontrar uma resolução, seja esta individual ou cooperativa. A proposta dos modelos não é reproduzir o mundo real, é notadamente impossível expor a complexidade do mundo real dentro de um modelo. Porém, o objetivo é procurar entender algumas coisas sobre o comportamento do sistema e também entender os efeitos e mudanças ocorridas sobre este, em respostas as mudanças.

Assume-se o objetivo de criar e usar modelos porque muitas situações do mundo real são complexas e geralmente não podem ser entendidas sem algum tipo de experimentação controlada. Em outros casos isto não é possível pela dificuldade de repetição de certos fenômenos, pelos custos associados, ou pelo risco que pode representar a seus observadores e/ou observados. Através dos modelos é possível capturar a essência dos sistemas do mundo real e, em muitos casos, é possível fornecer uma acurada previsão dos acontecimentos. Um tipo de modelo atualmente em evidência é o de simulação em computador.

Um modelo de simulação, de modo geral, consiste em reproduzir artificialmente uma situação ou fenômeno natural. Os modelos de simulação se baseiam na idéia de que os programas exibem o comportamento do sistema real e desta forma, ao transportar um fenômeno do mundo real para um artificial, na qual hipóteses particulares podem ser exploradas, possibilita o processo de resolução de problema como um ambiente de aprendizagem. A simulação pode ser vista repetindo experimentos de maneira análoga à que se faz num laboratório real. 
Numa gama de problemas que poderíamos modelar, daremos atenção especial àqueles incorporados aos chamados sistemas complexos. Sistemas complexos são aqueles cujos fenômenos e comportamentos surgem de interações de partes simples (Ruthen, 1993). São exemplos de sistemas complexos: uma colônia de formigas, mercado financeiro, uma cidade, bando de pássaros voando, reações do sistema imunológico, congestionamento de trânsito ou o funcionamento do cérebro humano. Em todos esses sistemas não há controle centralizado e os modelos são determinados pelas interações locais entre os componentes descentralizados (Resnick, 1991). Todos esses sistemas podem ser modelados com a composição de muitas partes distribuídas interagindo entre si e com o ambiente.

Os problemas referentes a sistemas complexos chamaram a nossa atenção pelo fato de estar presente em nossas vidas e permear as disciplinas tradicionais do currículo educacional. Apesar de raramente ser encontrado nas disciplinas até o ensino médio, possivelmente pelo seu caráter não-linear. E realmente, muitos estudos têm mostrado que tanto no público em geral quanto nas disciplinas de ciências não é fácil encontrar bons pensadores sobre sistemas onde um conjunto de agentes autônomos interage (Resnick, 1991).

A proposta apresentada neste artigo é resultado do estudo de várias metodologias de resolução de problemas e resolução cooperativa de problemas que serviram de suporte para a concepção do "AProSiMA - um ambiente para resolução cooperativa de problemas baseado em simulação multiagente", tendo como componente o NetPlay, que é uma ferramenta para construção de modelos de simulação baseada em multiagente, no domínio de sistemas complexos. Este artigo está organizado da seguinte forma: Resolução de problemas, tópico 2, apresenta um breve retrospecto dos métodos de resolução de problemas. Em seguida apresentamos a abordagem referente à resolução cooperativa de problemas, tópico 3. Os ambientes de apoio ao trabalho cooperativo na internet e avaliação dos mesmos com relação à utilização como ambientes de resolução cooperativa de problemas é apresentado no tópico 4. Por fim apresentamos, no tópico 5, o AProSiMA - ambiente para resolução cooperativa de problemas baseado em simulação multiagente, seguido de nossas considerações finais.

\section{Resolução de problemas}

A habilidade em resolver problemas vai muito além da simples aplicação de conhecimentos e regras. Resolver problemas implica no desenvolvimento de estratégias cognitivas flexíveis que ajudam a extrair dados inesperados de situações, algumas vezes, mal estruturadas para produzir soluções significativas. A resolução de problemas é importante em muitos campos, tanto de domínio específico quanto genérico. Métodos de resolução de problemas têm sido desenvolvidos há muitos anos. Encontramos em (Deek, 1997), um retrospecto sobre os métodos de resolução de problema passando desde o método cientifico de Dewey (1910); a visão criativa do método não sistemático de Wallas (1926); o trabalho de Polya (1945) que faz a junção dessas duas abordagens e destaca a heurística; os métodos de Kingsley e Garry (1957) que é uma variação do método científico de Dewey; o método de Johnson (1955) refere-se ao método criativo de Wallas; Osborn (1953) que introduz a abordagem de resolução de problemas criativos (creative problem solving - CPS); trabalho seguido por Parnes (1967); Simon (1960) que viu o processo de resolução de problemas como quatro capacidades cognitivas: inteligência, projeto, escolha e implementação; até o método Dual Commom Model (DCM) desenvolvido por Deek (1997), que integra a metodologia de resolução de problemas e a tarefa de desenvolvimento de programa computacional com a 
habilidade e conhecimento cognitivo necessário a cada estágio do processo de resolução.

Métodos mais recentes foram desenvolvidos visando auxiliar os estudantes de matemática, ciências e engenharia. Esses surgiram derivados dos já mencionados, muitos deles baseados no modelo de Polya, sem, no entanto apresentarem mudanças substanciais. Geralmente, esses métodos são apresentados variando em quantidade de etapas e detalhes, mas todos incorporando uma progressão sobre a identificação do problema, levantamento de hipóteses, elaboração de um plano, execução do plano e checagem da solução, entre eles (Schoenfeld, 1985, 1990), (Kaufman, 1988), (Etter, 1995), (Hartman, 1996), (Deek, McHugh, Turoff, 2000).

Observando a proposta de vários teoristas sobre métodos de resolução de problemas, percebe-se que em sua essência trata de um conjunto encadeado de passos lógicos que conduzem o resolvedor a identificar o problema e, por meio de elaboração e execução de estratégias, chegarem a uma solução que deverá ser validada. Um aspecto importante nos métodos de resolução de problema é que eles variam em atividades de coleta, planejamento e análise de dados. Normalmente, a compreensão de um problema requer a identificação dos dados oferecidos, das restrições, das condições em que ocorrem e das relações entre elas. Invariavelmente, a resolução de um problema requer alguma forma de partição do problema, isto é, divisão em problemas menores.

\section{Resolução cooperativa de problemas}

Muitos modelos de resolução cooperativa de problemas são baseados nos métodos de resolução individual. Esse modelo inclui não apenas passos de resolução de problemas genéricos, tarefas de domínio específico, e requisitos de habilidades cognitivas, mas também a comunicação e a coordenação das atividades requeridas pelo ambiente cooperativo (Deek, McHugh, Turoff, 2000). Um modelo clássico de resolução de problema cooperativo desenvolvido por Simon (1960) requer: a) desenvolvimento de plano - um plano de comportamento para o grupo é desenvolvido pra todos os membros do grupo, não um plano individual pra cada membro; b) comunicação do plano - esse plano é efetivamente comunicado para todos os membros do grupo; d) modificação do comportamento - cada membro individual do grupo deve concordar com o planejado, no sentido do acordo permitir que seu comportamento direcione ao planejado.

O objetivo desse acordo contratual sobre o planejamento do grupo é para avaliar os membros individuais sobre as tarefas e para predizer o comportamento dos outros membros. Esse processo é interativo e ocorre de acordo as exigências do projeto de trabalho. A resolução de problemas num ambiente cooperativo, conforme observado por Hohmann (1997, apud Tommarello, 1992), difere substancialmente do modo que um indivíduo aplicaria esse mesmo método. Há muitos pontos a serem observados no trabalho cooperativo em relação à comunicação e as relações entre os membros do grupo, sendo um importante aspecto a forma como o indivíduo interage. Grupos cooperativos parecem capazes de tratar com tarefas complexas mais eficientemente do que um indivíduo apenas, particularmente porque um grupo terá uma ampla gama de habilidades e capacidades mais do que um indivíduo (Finnegan, O’Mahony, 1996). Em um grupo, os membros envolvidos são afetados pela cooperaçao, porque um indivíduo aprende as habilidades e capacidades de outro membro do grupo.

Como apresentado acima, a busca para a resolução de problemas envolve abordagem interdisciplinar e a participação cooperativa. Em geral, os especialistas em um domínio específico encontram-se geograficamente distribuídos, o que impõe dificuldades à cooperação. $\mathrm{O}$ advento e a atual expansão da World Wide Web ao 
oferecer um suporte de comunicação e tratamento à distância da informação, aproxima os especialistas facilitando o estabelecimento de equipes de trabalho para a resolução cooperativa de problemas.

\section{Ambientes de apoio ao trabalho cooperativo na internet}

Apresentamos uma revisão bibliográfica, não exaustiva, dos ambientes que suportam atividades cooperativas na internet. Existem vários ambientes que apóiam o trabalho/aprendizagem cooperativa por computador, entre eles destacamos, em ordem alfabética: AmCorA, Belvedere, CaMILE, Groove (atual Groove Virtual Office), GroupSystem, HM-Card, Tango Interactive.

Todos os ambientes visitados oferecem as ferramentas básicas de interação síncrona e assíncrona, segundo sua finalidade de projeto. Entretanto, alguns deles disponibilizam ferramentas mais apropriadas para o apoio à resolução de problemas. $\mathrm{O}$ Lotus Note fornece assistência à coordenação do grupo, mas não fornece ferramentas específicas para atividades de cooperação em tempo real ou assíncrona. As características apresentadas pelo Groove facilitam o desempenho do grupo nos principais aspectos do processo de resolução de problemas. As tarefas podem ser facilmente identificadas, distribuídas, comunicadas e modificadas. A sua ferramenta de brainstorming permite aos usuários apresentar e discutir as diferentes idéias, porém não possibilita a votação para escolha. O GroupSystem é um excelente ambiente com ferramentas colaborativas. Seu principal objetivo é apoiar as atividades de conhecimento colaborativo, semelhante ao planejamento estratégico e análise de risco. Oferece uma ferramenta de brainstorming associada com uma ferramenta de votação, requerido na resolução de problema para o processo de decisão. Aliado a ferramenta de whiteboard o sistema oferece ainda uma ferramenta adicional - paint, que possibilita o usuário esboçar e editar imagens no whiteboard, que podem ser impressas ou salvas em arquivo.

Vários outros aspectos e ferramentas poderiam ser destacados, uma vez que a resolução cooperativa de problemas requer direção e apoio, que inclui o esforço para melhorar as atividades cognitivas do grupo enquanto engajados na resolução cooperativa de problemas, que esses sistemas não incorporam. A utilização desses ambientes para apoiar a aprendizagem cooperativa deixa a desejar em vários aspectos, entre eles, na ausência de uma metodologia de resolução de problemas que auxilie o grupo a estabelecer estratégias e heurísticas para a resolução de problemas. Outro fator é a inexistência de uma ferramenta para construção de modelos de simulação que, como afirmado anteriormente, é uma forma de representar, explicar e entender os fenômenos em estudo, objetivando encontrar uma resolução seja esta individual ou cooperativa.

Objetivando a aprendizagem cooperativa concebemos o AProSiMA - Um ambiente para resolução cooperativa de problemas baseado em simulação multiagente, com características para web, pela facilidade de uso e acesso por meio de navegadores disponíveis nas máquinas dos usuários, e composto por várias ferramentas para apoiar o processo de resolução cooperativa de problemas, entre elas uma para construção de modelos de simulação baseado em multiagente - NetPlay.

\section{AProSiMA - arcabouço conceitual}

Na busca por estratégias de soluções criativas para resolução cooperativa de problemas, uma alternativa que tem se mostrado útil é a criação de modelos de simulação por computador. Através do modelo de simulação é possível ter uma visão geral sobre o 
problema em estudo, fazer conjecturas sobre as variáveis que interferem no problema, testar possibilidades e avaliar as conseqüências de tomada de decisão.

Um modelo de simulação, de modo geral, consiste em reproduzir artificialmente uma situação ou fenômeno natural. O processo de simulação liga situações e problemas com ambientes de software através de modelos. Esses modelos se baseiam na idéia de que os programas exibem o comportamento do sistema real, isto é, transportando o fenômeno do mundo real para um artificial, na qual hipóteses podem ser exploradas, possibilitando o processo de resolução cooperativa de problema como um ambiente de aprendizagem. Construir um modelo apropriado para realizar a simulação é crucial (Biehler, 1991 apud Sanchez, Canal, 2001,). Podemos afirmar então que a resolução cooperativa de problemas é essencial no desenvolvimento da modelagem de simulação e vice-versa. Além disso, muitas tarefas básicas que dão à estrutura da modelagem de simulação podem ser vistos como subproblemas no processo de resolução de problemas: compreender o problema, verificar os dados existentes, estabelecer um plano de ação, executar o plano, validar os dados e fazer um retrospecto da resolução completa (Polya, 1995). Quanto mais completo e sofisticado for o modelo a ser simulado, maior será o número de relações que coexistem simultaneamente, fazendo com que um fator adicional cresça em importância: a cooperação.

Para se ter um modelo de simulação que represente um problema a ser solucionado é necessário todo um trabalho de resolução cooperativa, pois o crescente número de relações e o conhecimento em domínio específico têm necessitado o uso de equipes ou grupos para desenvolver estratégias e soluções.

Com o objetivo de contribuir para o desenvolvimento deste campo de pesquisa, apresentamos neste trabalho um ambiente projetado para a resolução cooperativa de problemas e desenvolvimento de modelos de simulação, conforme apresentado na Figura 1.

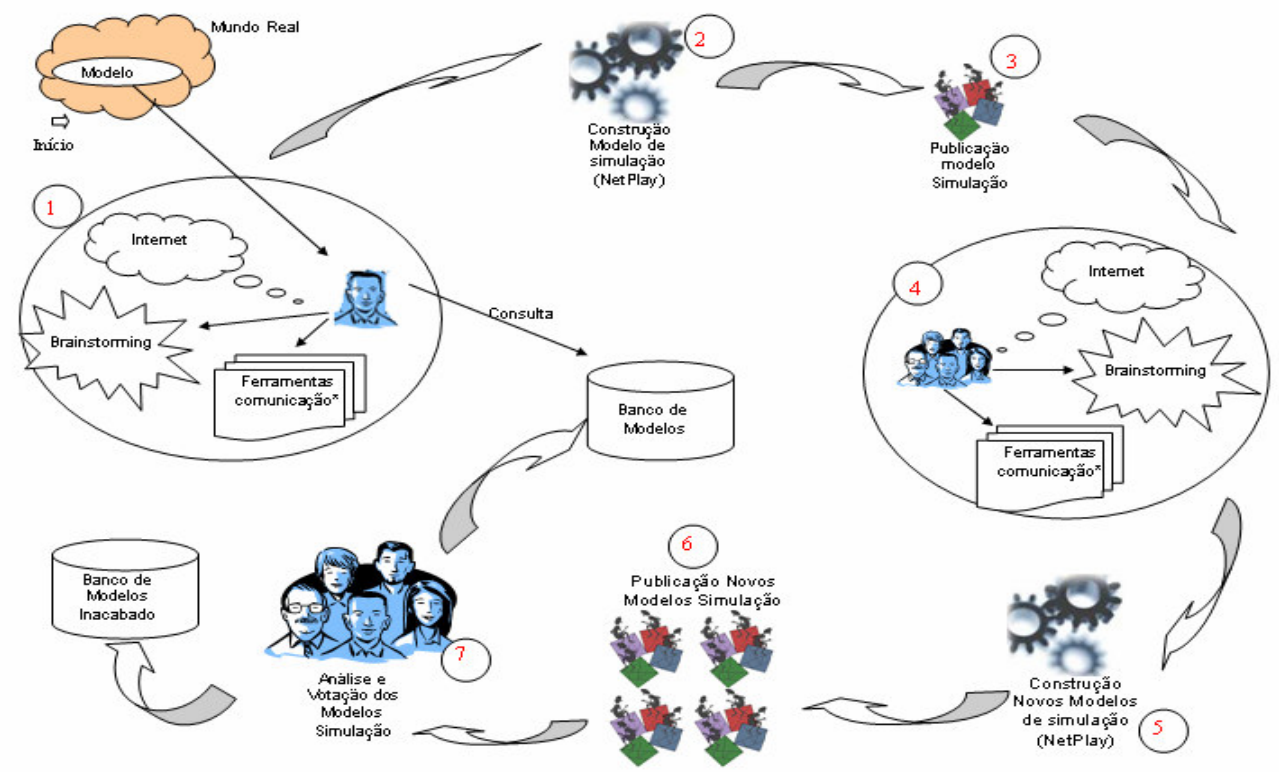

Figura 1 - AProSiMA - Ambiente para resolução cooperativa de problemas baseado em simulação Multiagente

\subsection{Descrição do ambiente}

Quando observamos um sistema um dos objetivos é tentar compreende-lo, isto é, criar uma teoria que represente esse sistema como um todo, independente do comportamento 
de suas partes. Para que isso ocorra é necessário analisá-lo cuidadosamente, buscando regularidades, padrões de comportamento, relação entre as interações, entre outros.

Uma vez que é muito difícil capturar todos os dados de um sistema real para reproduzir uma sociedade artificial, reforçamos, mais uma vez, a necessidade do trabalho cooperativo. Usando as ferramentas groupware para apoio ao trabalho cooperativo apoiado por computador e uma ferramenta de modelagem de simulação, apresentamos o "AProSiMA - Ambiente para resolução cooperativa de problemas baseado em simulação multiagente", Figura 1. Neste ambiente os usuários constroem modelos, que são representações extraídas do mundo real, auxiliados pelo processo de modelagem e resolução cooperativa. Procuramos representar o processo de criação/resolução cooperativa de problema usando o modelo de simulação suportada pelo ambiente "AProSiMA".

O ponto de partida. Em geral, o usuário se defronta com um problema do mundo real e, a partir daí, procura representá-lo através de um modelo de simulação para ganhar entendimento sobre o mesmo. No início do processo de construção do modelo é usada a ferramenta de brainstorming disponível (momento 1, Figura 1). Após ganhar algum entendimento sobre o modelo, o usuário passa para a ferramenta de simulação disponível no ambiente - Netplay (ferramenta já desenvolvida pelos autores). Esta possibilita a ele construir o seu próprio modelo de simulação (momento 2, Figura 1), tendo como orientação o processo de resolução de problema a ser apresentado na Figura 2. Após construído a primeira versão do modelo de simulação o usuário deve publicá-lo (momento 3, da mesma figura) para que os demais participantes do ambiente possam analisá-lo, inferir sobre ele e apresentar novas versões, num trabalho cooperativo que resultará numa melhor abrangência do modelo (momento 4 e 5, Figura 1). Depois, numa sessão conjunta, discutir os procedimentos para efetivação da idéia. Esse processo é acompanhado sistematicamente pelas publicações das contribuições realizadas pelos cooperadores (momento 6, Figura 1). Periodicamente, usando uma ferramenta de votação, os participantes do ambiente votam os modelos apresentados, classificando-os com relação a maior proximidade com o objetivo a ser simulado (momento 7, Figura 1). Os demais modelos são arquivados num "banco de modelos incompletos", pois poderão dar origem a soluções de outros problemas. Os modelos desenvolvidos são armazenados num "banco de modelos", servindo de referência para novos problemas, conforme pode ser observado na Figura 1. Todo esse processo é contínuo, uma vez que o interesse por um problema não acaba quando encontramos uma solução, é preciso buscar outras soluções, analisar suas propriedades, buscar generalizações etc.

As ferramentas de comunicação possibilitarão aos usuários traçar detalhes das idéias sobre o modelo com os demais membros envolvidos no projeto. A ferramenta brainstorming possibilitará a equipe (ou o próprio modelador) esboçar as idéias numa estrutura hierárquica. As idéias desenvolvidas com esta ferramenta poderão ser organizadas e armazenadas juntamente com o modelo construído. O processo de construção do modelo, bem como as discussões sobre sua modelagem será acompanhado através de uma ferramenta de publicação colaborativa - Wiki.

A Figura 2 mostra que a atividade de construção é particularmente influenciada por dois aspectos: as metas para a construção do modelo e a simulação propriamente dita. O modelador define as solicitações com relação ao que deve ser capturado pelo modelo, depois atribui às restrições com relação a como essas coisas podem ser representadas dentro do modelo. Assim, quando olhamos para um modelo procurando mais detalhes, podemos identificar um ciclo de atividades com duas principais tarefas distintas. 
Geralmente, quando iniciamos o processo de construção de um modelo, o modelador analisa a tarefa (metas ou o problema), a seguir aplica seu conhecimento no domínio do problema. Certamente, se o modelador não possui domínio do conhecimento sobre o problema, ele deve buscar tal conhecimento antes de iniciar o processo de modelagem. O ambiente AProSiMA oferece recursos para a busca de informações e/ou disponibilizar o problema para que outros usuários do ambiente possam cooperar (momento 1 e 3, Figura 1). O fenômeno do mundo real deve ser então identificado, selecionado e abstraído dentro de um conjunto de características capturadas dentro do modelo. Essas características devem ser mapeadas como elementos componentes do modelo que satisfazem às entradas da ferramenta de simulação. Todas essas atividades não são independentes. Conforme indica a Figura 2, o processo de modelagem é cíclico, cada elemento deve ser tratado como importante para determinar o resultado no processo de construção do modelo.

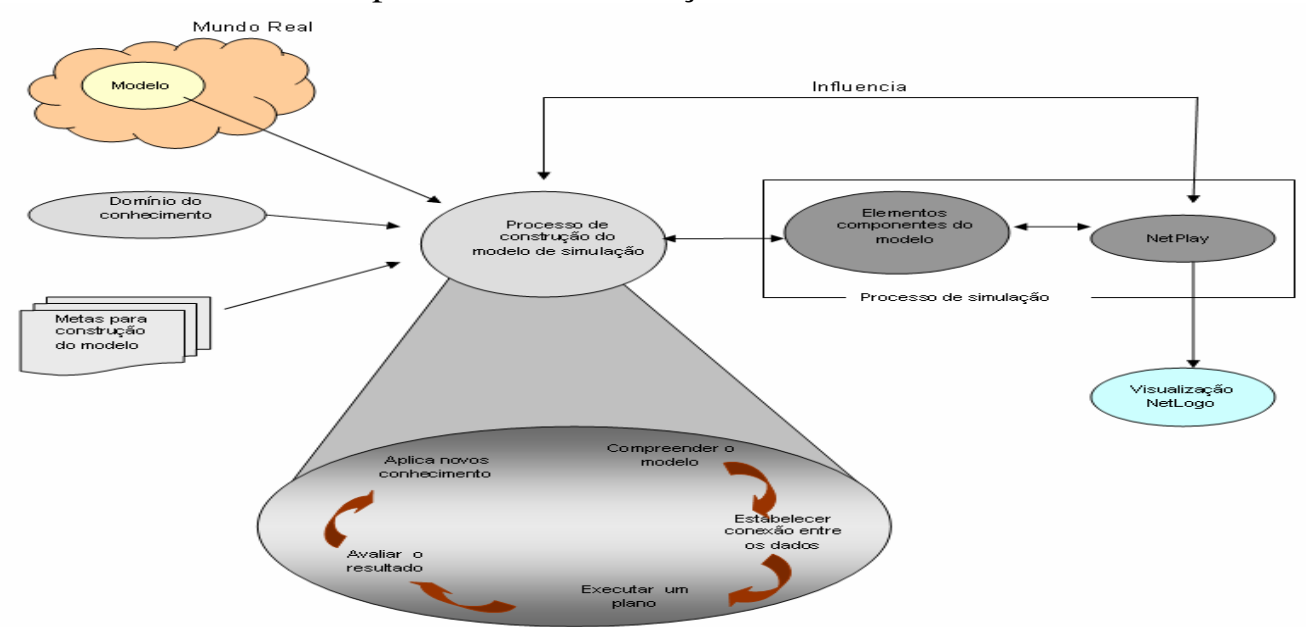

Figura 2 - Processo de modelagem de problema para descrever o modelo de simulação

O processo de modelagem descrito, Figura 2, segue o modelo de resolução de problemas apresentado por Polya (Polya, 1995): a) compreensão do problema (modelo) - o modelador deve procurar compreender o modelo (metas para construção do modelo) e a partir daí selecionar e abstrair elementos do fenômeno a ser modelado, procurar compreender as relações e como ocorrem as interações entre os objetos (agentes) no modelo (domínio do conhecimento); b) estabelecimento de um plano - buscar modelos correlatos, reformular o modelo, associar os elementos componentes do modelo a algum modelo semelhante, procure fragmentar o problema e repita o processo, leve em consideração todos os elementos associado ao modelo; c) execução do plano - definida as relações entre os elementos (agentes), componha as regras de interação entre os agentes e demais componentes do modelo de simulação; d) avaliação dos resultados execute a simulação e análise os resultados obtidos.

\subsection{NetPlay - ferramenta para construção do modelo de simulação}

A ferramenta NetPlay é uma componente do ambiente AproSiMA (momento 2, Figura 1), projetada para a construção de modelos de simulação, que são representações extraídas do mundo real, na perspectiva do uso da modelagem multiagente, da capacidade de reuso dos objetos projetados e visando diminuir o esforço da programação, para que estes possam visualizar o comportamento resultante das interações definidas e fazer conjecturas em relação ao modelo e tomada de decisão. 
Como já mencionado, trabalharemos com a construção de modelos no âmbito dos sistemas complexos.

A arquitetura da ferramenta pode ser acompanhada pela Figura 3. A linguagem adotada pela ferramenta segue o paradigma visual, sendo a programação realizada através da composição de regras simples de comportamento, manipulada através de figuras ilustrativas e caixas de diálogo. Desta forma não se visualiza código em forma de texto. O ambiente de desenvolvimento é onde o modelador escreve o programa, isto é, onde ele define os componentes do modelo: agentes (que são as classes) as regras de comportamento (que são os métodos) e as características de todos os agentes (que são os atributos).

Para definir uma sociedade de agentes é necessário dispor de elementos que descrevam essas sociedades. Em sua arquitetura o NetPlay disponibiliza esses elementos. Num primeiro momento são estabelecidos: definição de agentes (cor, forma, tamanho, quantidade, identificação), definição de variáveis (referentes aos agentes ou especificas ao fenômeno), posicionamento dos agentes no ambiente e estabelecimento das regras de comportamento (tipo de movimento, ações). Na seqüência, definido o comportamento individual dos agentes, passa-se a fase de composição das regras que definem as interações entre agentes e entre agente e ambiente. O passo seguinte é a geração automática do código que possibilita a visualização do modelo de simulação, através de um applet ou no ambiente Netlogo (Wilensky, 1999), através da exportação do código gerado. Todos os modelos são armazenados no banco de dados do ambiente. Todas as ações resultantes na visualização são descritas na ferramenta NetPlay.

Associado a ferramenta de construção de modelos encontra-se o editor de versões, um componente que tem como objetivo propiciar a participação cooperativa para desenvolvimento do modelo, Figura 4. Durante o desenvolvimento de um modelo, pelo grupo de usuários, cada elemento do grupo pode fazer as suas considerações sobre o modelo, criando assim um encadeamento de sugestões (versões do modelo) que numa sessão posterior será votada para definir quais considerações comporão a versão final do modelo. Mais de uma versão pode ser aprovada e lançada ao banco de modelos. $\mathrm{O}$ objetivo é compor uma biblioteca o mais abrangente possível para que os aprendizes possam usufruir do seu conteúdo. Outro aspecto é a facilidade de reuso e adaptabilidade dos modelos já apresentados.

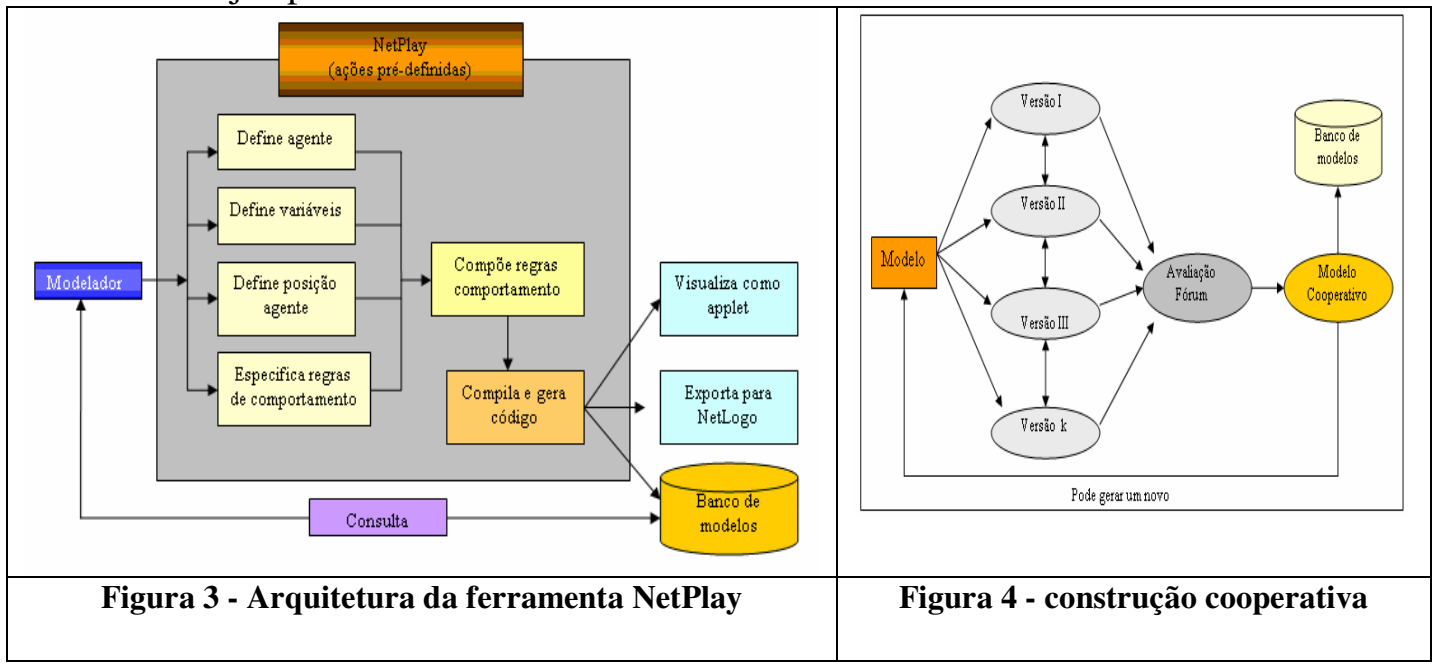

\subsection{Ferramentas adicionais}

A ferramenta NetPlay, já implementada, tem possibilitado descrever modelos que apresentam comportamento semelhante aos desenvolvidos em ambientes similares. 
Todas as facilidades disponíveis no ambiente NetLogo (Wilensky, 1999), foram incorporadas ao NetPlay para o completo desenvolvimento do modelo. Entretanto, percebemos que da forma como os elementos são plotados no gráfico não oferecem precisão para análise. Isto ocorre pelo fato do processamento gerar muitos dados de saída, que resulta na necessidade de redução da escala do gráfico. Além do mais, para modelos com variações esparsas, há um número excessivo de dados desnecessários. Assim, como trabalho futuro e para auxiliar no processo de resolução cooperativa de problemas pretendemos disponibilizar algumas outras ferramentas no ambiente que venham favorecer as duas etapas: a construção e a análise do modelo. Para a primeira, pretendemos disponibilizar uma ferramenta compartilhada, para utilização anterior ao processo de desenvolvimento do modelo, para construção virtual de esquemas de solução, cujo propósito é auxiliar os desenvolvedores a esquematizar a sequiência de ações que descrevem o comportamento do modelo, os elementos que o compõe e o encaminhamento resultante das ações. Para a segunda, uma ferramenta que exporte, para um arquivo externo, os dados plotados no gráfico, porém dispondo de um mecanismo de monitoramento das variações de modo que: a) registre apenas os valores nos intervalos de transição; b) acompanhe o comportamento das variáveis e registre os intervalos onde houver interseção; c) disponibilize um mecanismo de busca para esses dados.

\section{Considerações finais}

Analisar os problemas à luz de modelos de simulação baseado em multiagente oferece uma gama de questionamentos, às vezes imperceptíveis quando tratados por uma outra abordagem, e esses devem ser apoiados e explorados. A aprendizagem através de resolução de problemas não significa apenas encontrar uma resposta que satisfaça a uma questão proposta, mas sim procurar entender o sistema como um todo. É importante, além disso, supor novas situações e analisar as conseqüências advindas destas. Para isso é necessário supor novos valores para as variáveis e analisar o comportamento do modelo. Podemos explorar o modelo, atribuindo diferentes valores às suas variáveis livres e assim poder avaliar seus efeitos sobre o sistema. Para aprender com o modelo de simulação é preciso exploração, necessitando para isso de algumas ferramentas: um quadro visual do sistema em funcionamento (palco de interações), monitores exibindo valores correntes para variáveis de saída e gráfico mostrando, em tempo real, a variação de algumas variáveis.

Para construir um modelo artificial, representante de um sistema complexo, que exiba o comportamento do sistema real, geralmente, é necessário envolver uma grande quantidade de variáveis e descrever detalhes de seu comportamento. A captura de todos esses elementos já caracteriza um processo de resolução de problemas que necessita de cooperação. Isto posto, fortalece nosso empenho em projetar um ambiente que apóie a aprendizagem através de resolução cooperativa de problemas, fazendo uso da ferramenta de construção de modelos de simulação, conforme o AProSiMA, aqui apresentado. Como trabalhos futuros destacamos o empenho na implementação das demais ferramentas que compõem o AProSiMA: a) gerenciador de modelos ferramenta que relaciona modelos, pessoas e informações referentes ao problema a ser resolvido; b) esquematizador - ferramenta que possibilita aos usuários, cooperativamente, discutir o fenômeno e estabelecer diretrizes para a construção do modelo (flowchart); c) ferramentas de comunicação - possibilita a interação entre os usuários do sistema para a resolução cooperativa de problemas (chat, e-mail, wiki, fórum). Entretanto, o próximo passo da pesquisa é o oferecimento de duas disciplinas, 
uma na graduação (ciência da computação) e outra na pós-graduação (mestrado em informática) no Departamento de Informática da UFES - "Simulação baseada em Sistemas Multiagentes - Aplicações em Educação". Nesta disciplina faremos uso do NetPlay como ferramenta para construção de modelos com os objetivos de: a) obter dados que nos permita aprimorar os ambientes; b) iniciar um processo de sensibilização para o estudo de sistemas complexos e c) analisar os modelos e o processo de resolução de problemas, bem como as conseqüências das tomada de decisão.

\section{Referência bibliográfica}

DEEK, F., MCHUGH, J., TUROFF, M., "Problem Solving and Cognitive Foundations for Program Development: An Integrated Model", submitted for review to the Journal of Cognitive Science, 2000

DEEK, F.P., An Integrated Environment For Problem Solving and Program Development, Unpublished Ph.D. Dissertation, New J. Institute of Technology, 1997.

FINNEGAN, P., O'MAHONY, L., “Group Problem Solving and Decision Making: an Investigation of the Process and the Supporting Technology", Journal of Information Technology, Vol. 11, Num. 3, September 1996

POLYA, George, A arte de resolver problemas: um novo aspecto do método matemático; trad. Heitor Lisboa de Araújo, $2^{\mathrm{a}}$ reimpressão, Ed. Interciência, RJ 196p.

RESNICK, M., Turtles, termites, and traffic jams: explorations in massively parallel microwords, The MIT Press, Massachusetts, USA, 1991.

RUTHEN, R., Adapting to Complexity. Journal: Scientific American, 268, 1993.

SÁNCHEZ, E. S., CANAL, G. Y., Computational Simulation and Conditional Probability Problem Solving, http://www.stat.auckland.ac.nz/ iase/publications/3/3638.pdf

SANTORO, F. M., BORGES, M. R. S., SANTOS, N., Um Framework para Estudo de Ambientes de Suporte à Aprendizagem Cooperativa, Sbie 1998

SIMON, H. A., The New Science of Management, Harper and Row, New York, 1960.

TOMMARELLO, Joanna DeFranco, Literature review of collaborative problem solving and groupware in the software development domain, State of the Art Review Paper, 1992

WILENSKY, U., NetLogo. http://ccl.northwestern.edu/netlogo. Center for Connected Learning and Computer-Based Modeling. Northwestern University, Evanston, IL., 1999 
${ }^{\mathrm{i}}$ AmCora (www.gaia.ufes.br/amcora/), Belvedere (http://advlearn.lrdc.pitt.edu/belvedere/), Camile (http://www.cc.gatech.edu/gvu/edtech/CaMILE.html),

Groove (http://www.groove.net/home/index.cfm), Goup-system (www.groupsystems.com), HM-Card (Santoro et al, 1998), Tango (http://www.tangointeractive.com/) 